\title{
Utilidad de la colangiografía transoperatoria en pacientes con diagnóstico de coledocolitiasis con estudio de imagen 0 endoscópico preoperatorio
}

\author{
Use of intraoperative cholangiography in patients diagnosed with choledocholithiasis \\ with preoperative imaging or endoscopic study
}

\author{
Juan J. Díaz-Osuna ${ }^{*}$, Adolfo Leyva-Alvizo y Carlos E. Chávez-Pérez²
}

${ }^{1}$ Servicio de Cirugía General; ${ }^{2}$ Servicio de Medicina del Enfermo en Estado Crítico. Hospital San José TecSalud, Monterrey, Nuevo León, México

\begin{abstract}
Resumen
Antecedentes: La colecistectomía es uno de los procedimientos quirúrgicos más realizados. Muchos cirujanos defienden la colangiografía transoperatoria (CTO) sistemática, pero su uso es cada vez menos frecuente por la disponibilidad de otras herramientas de estudio empleadas preoperatoriamente que pueden obviar este procedimiento. Objetivo: Valorar la utilidad de la CTO cuando se cuenta con un estudio de imagen o endoscópico preoperatorio. Analizar la relación entre el reingreso por un cuadro obstructivo de vía biliar y el abordaje al que se sometió el paciente. Método: Estudio retrospectivo y comparativo de 117 pacientes ingresados por coledocolitiasis en un periodo de 2 años. Las variables analizadas fueron demográficas, diagnóstico, CTO, estudio previo y reingreso. Se incluyeron pacientes mayores de 18 años con diagnóstico de coledocolitiasis en quienes se realizó colecistectomía. El análisis estadístico se hizo comparando grupos mediante la prueba de ji al cuadradocon una significancia estadística de $p<0.05$. Resultados: El grupo con estudio preoperatorio y CTO no presentó reingresos. Hubo nueve reingresos, siendo el diagnóstico de lito residual. Los grupos sin CTO presentaron más reingresos. El grupo con mayor número de reingresos fue el que no se realizó CTO y no contaba con estudio preoperatorio. No se reportó mortalidad. Conclusión: Los grupos en los que se realizó CTO presentaron menos reingresos por lito residual.
\end{abstract}

PALABRAS CLAVE: Colangiografía. Colecistectomía. Coledocolitiasis. Colangiopancreatografía retrógrada endoscópica. Biliar.

\begin{abstract}
Background: Cholecystectomy is one of the most performed procedures worldwide. Many surgeons defend the use of routine intraoperative cholangiography (IOC), but this action is getting less practiced because other preoperative tools that can omit IOC. Objective: Evaluate the utility of performing intraoperative cholangiography in patients whom got a preoperative imaging or endoscopy study. We analized the association between the diagnostic-therapeutic approach and hospital readmissions because of bile duct obstruction. Method: Retrospective and comparative study of 117 patients admitted with choledocholithiasis and submitted to cholecystectomy between January 2014 and December 2016. The statistical analysis was performed comparing groups using chi squared test with a statistical significance of $p<0.05$. Results: Patients whom got a preoperative study and had performed an intraoperative cholangiography didn't readmitted. The study reported nine readmissions, being residual gallbladder stone the diagnostic. Patients whom didn't get an intraoperative cholangiography reported the most read-
\end{abstract}

\footnotetext{
Correspondencia:

*Juan J. Díaz Osuna

Avda. Morones Prieto, 3000

Col. Los Doctores

C.P. 64710, Monterrey, N.L., México

E-mail: jjaime.diaz@ hotmail.com
}

Fecha de recepción: 18-05-2018

Fecha de aceptación: 28-07-2018

DOI: $10.24875 / C I R U .18000468$

Cir Cir. 2019;87:146-150

Contents available at PubMed www.cirugiaycirujanos.com 
missions. The studied group who registered the most readmissions was the one who didn't get performed an intraoperative cholangiography nor a preoperative study. No mortality was reported. Conclusion: Patients who had IOC performed reported less readmissions for residual stone.

KEY WORDS: Cholangiography. Cholecystectomy. Endoscopic retrograde cholangiopancreatography. Choledocholithiasis. Bile.

\section{Introducción}

La colangiografía transoperatoria (СTO) es una prueba diagnóstica que se realiza durante el procedimiento quirúrgico para observar la morfología de la vía biliar con rayos $\mathrm{X}$ o fluoroscopía dinámica'.

El objetivo principal de la CTO es identificar litos en la vía biliar, ver la anatomía biliar y prevenir lesiones de la vía biliar². La CTO tiene sus indicaciones, tanto preoperatorias como transoperatorias ${ }^{3}$. Entre las preoperatorias se incluyen ictericia, antecedente de pancreatitis biliar, elevación de enzimas hepáticas y dilatación de la vía biliar por imagen. Entre las transoperatorias se incluyen dilatación de la vía biliar extrahepática, poca claridad anatómica y lesión de la vía biliar . No existen guías que especifiquen si realizar СTO de manera sistemática o selectivamente ${ }^{5}$, sino que esto es a consideración del cirujano, siempre teniendo en cuenta el cuadro clínico del paciente y las indicaciones para realizar CTO. Durante la popularización de la colecistectomía laparoscópica en los años 1990 se recomendaba la CTO sistemática como parte de protocolo de cirugía segura ${ }^{6}$, pero la tendencia actual es realizar СТО selectiva.

La incidencia de coledocolitiasis sin manifestaciones clínicas es del 5.2-12\%7. Los grupos de cirujanos que realizan CTO sistemática reportan una incidencia de un $4.6 \%$ de litos en la vía biliar en pacientes asintomáticos sin antecedente de pancreatitis biliar ${ }^{8}$. El paso espontáneo de un lito en la vía biliar al duodeno está documentado, y los estudios reportan una incidencia de hasta el $75 \%$ en pacientes con pancreatitis de origen biliar y del $55 \%$ en pacientes con datos clínicos de coledocolitiasis 9 . El índice de lito residual en los pacientes en que se realizó CTO sin evidencia de obstrucción es inferior al 1\% ${ }^{9}$.

El uso de CTO puede reportar un $7-20 \%$ de falsos positivos, lo que lleva a una exploración de la vía biliar - colangiopancreatografía retrógrada endoscópica (CPRE) innecesaria, agregando morbilidad y costo, ya que se ha comprobado que dicho procedimiento aumenta los días de estancia hospitalaria ${ }^{10}$. El diagnóstico y el manejo de un lito residual puede ser un desafío para el cirujano. Hay diversas opciones de manejo para estos pacientes, entre las que se encuentran los abordajes endoscópico y quirúrgico.
El objetivo del presente estudio es valorar la utilidad de la CTO en pacientes con datos de coledocolitiasis que cuentan con estudio de imagen: Colangiopancreatografía por Resonancia Magnética (CRM) o Colangiopancreatografía Retrógrada Endoscópica (CPRE) realizado previo a la colecistectomía.

\section{Método}

Se trata de un estudio comparativo y retrospectivo, entre enero de 2014 y diciembre de 2016, realizado en dos unidades hospitalarias. Se incluyeron pacientes mayores de 18 años con diagnóstico de coledocolitiasis (por clínica, elevación de enzimas hepáticas o imagen) sometidos a colecistectomía. Se excluyeron aquellos en quienes no se realizó intervención quirúrgica o que tuvieran obstrucción extrínseca de la vía biliar, o expedientes incompletos. Las variables analizadas fueron la edad, el sexo, el diagnóstico, el estudio preoperatorio realizado (CRM), el manejo endoscópico (CPRE) y su reporte, las complicaciones transoperatorias, las conversiones a cirugía abierta, los hallazgos en la CTO, las complicaciones posoperatorias y la presencia de un nuevo cuadro obstructivo con reingreso por esta razón.

Los pacientes se dividieron en cuatro grupos conforme al manejo empleado:

- Grupo 1: CTO con estudio preoperatorio.

- Grupo 2: CTO sin estudio preoperatorio.

- Grupo 3: no CTO con estudio preoperatorio.

- Grupo 4: no CTO sin estudio preoperatorio.

Las decisiones sobre el manejo preoperatorio y transoperatorio del paciente con coledocolitiasis fue a criterio de los cirujanos, contando con equipo endoscópico y de imagen disponible para los grupos estudiados.

Se realizó el análisis estadístico mediante el programa IBM SPSS Statistics 22.0. La comparación entre grupos se llevó a cabo con la prueba de ji al cuadrado, y se obtuvo la razón de momios para determinar la fuerza de la asociación. Se otorgó una confiabilidad del $95 \%$ y se consideró $p<0.05$ como estadísticamente significativo.

El estudio fue aprobado por el Comité de Ética en Investigación de la Escuela de Medicina del Instituto Tecnológico y de Estudios Superiores de Monterrey 
Tabla 1. Características demográficas de la población estudiada. Se muestran el total y la división en pacientes en quienes se realizó (grupos 1 y 2) o no colangiografía transoperatoria (grupos 3 y 4), siendo similares los grupos

\begin{tabular}{|c|c|c|c|c|}
\hline Variable & $\begin{array}{c}\text { Total } \\
(n=117)\end{array}$ & $\begin{array}{c}\text { CTO } \\
(n=58)\end{array}$ & $\begin{array}{l}\operatorname{Sin} \text { CTO } \\
(n=59)\end{array}$ & $p$ \\
\hline \multicolumn{5}{|l|}{ Edad (años) } \\
\hline Rango & $22-94$ & $25-94$ & $22-75$ & NS \\
\hline Mediana & 47 & 47 & 46 & \\
\hline \multicolumn{5}{|c|}{$\begin{array}{l}\text { Distribución de } \\
\text { edades (años) }\end{array}$} \\
\hline $22-39$ & $36(30.76 \%)$ & $16(27.58 \%)$ & $18(30.50 \%)$ & NS \\
\hline $40-59$ & $47(40.17 \%)$ & 24 (41.37\%) & 25 (42.37\%) & NS \\
\hline 60 y más & $34(29.05 \%)$ & $18(31.03 \%)$ & $16(27.11 \%)$ & NS \\
\hline \multicolumn{5}{|l|}{ Sexo } \\
\hline Masculino & $36(30.76 \%)$ & $13(12.29 \%)$ & $23(18.18 \%)$ & 0.17 \\
\hline Femenino & $81(69.23 \%)$ & 45 (41.17\%) & 36 (28.34\%) & \\
\hline
\end{tabular}

CTO: colangiografía transoperatoria; NS: no significativo.

(CONBIOETICA-19-CEI-011-20161017) y por el Comité de Investigación de la Escuela de Medicina del Instituto Tecnológico y de Estudios Superiores de Monterrey (17 Cl 19039 003).

\section{Resultados}

Se revisaron 117 registros de pacientes que cumplieron con los criterios de inclusión para este estudio. Los grupos presentaron medianas y rangos de edad similares, y en cuanto a la distribución por edades ambos grupos presentaron porcentajes similares, sin diferencia estadísticamente significativa. Hubo una mayor prevalencia de pacientes de sexo femenino en este estudio ( $p=0.17$ ). El rango de edad con más pacientes fue 40-59 años (40.1\%) (Tabla 1).

En el grupo 1 hubo 37 pacientes, en el grupo 2 hubo 21 pacientes, en el grupo 3 hubo 54 pacientes y en el grupo 4 hubo 5 pacientes (Tabla 2).

El $71 \%$ de los pacientes contaban con estudio o manejo preoperatorio, siendo el de mayor uso la CPRE. El grupo 3 fue el que presentó más pacientes, siendo el abordaje más utilizado.

Se realizaron 74 CPRE, y de ellas, siete (9.45\%) CPRE preoperatorias con las que no se logró resolver el cuadro obstructivo. Estos pacientes se sometieron a exploración de la vía biliar, tres convencionales y cuatro laparoscópicas, de las que una se convirtió a cirugía abierta. No se registraron reingresos en estos casos. Se realizaron 67 CPRE que lograron resolver el cuadro obstructivo exitosamente mediante extracción de lito y esfinterotomía durante el mismo internamiento.

Los reingresos reportados fueron $9(7.69 \%)$. El diagnóstico de reingreso en todos los casos fue por lito residual. Los pacientes en quienes no se realizó СTO (grupos 3 y 4) fueron los que reportaron más casos de reingresos, en comparación con los pacientes en quienes se realizó CTO (grupos 1 y 2 ) $(p=0.0021)$ (Tabla 3$)$.

El grupo 3 contó con mayor muestra (46.15\%). En este grupo hubo tres reingresos, sin ser estadísticamente significativo frente al grupo 4 , que presentó la mayor tasa de reingresos $(p=0.034)$. No se reportó mortalidad en la muestra estudiada.

En el grupo 1 no se reportaron reingresos y en el grupo 2 hubo un reingreso que se resolvió mediante CPRE. En el grupo 3 se reportaron tres reingresos, que se resolvieron mediante CPRE.

El grupo 4 reportó reingresos en todos los pacientes $(100 \%)$. Fue el grupo que reportó más reingresos $(55.5 \%)$ del total, siendo estadísticamente significativo $(p=0.03)$. El cuadro obstructivo se resolvió mediante CPRE.

\section{Discusión}

La colelitiasis es un padecimiento muy común en la población occidental, con una incidencia estimada del $10-15 \%{ }^{11}$, y la colecistectomía por laparoscopia es el manejo estándar. La presencia o la sospecha de coledocolitiasis hacen que el clínico se vea ante diversas opciones para resolver la patología. En este estudio se analizaron distintos tipos de decisiones preoperatorias y de tratamiento, así como los resultados en cuanto a reingresos asociados a lito residual.

Los pacientes de este estudio contaban con diagnóstico de coledocolitiasis por clínica (ictericia), exámenes de laboratorio (elevación de enzimas hepáticas) o imagen (estudio reportando dilatación de la vía biliar o evidencia de lito en la vía biliar). Se decidió realizar CPRE cuando los pacientes cumplían los criterios de sospecha de coledocolitiasis. Se realizó esfinterotomía durante las CPRE realizadas en este estudio.

Hay distintos métodos por los cuales puede estudiarse la patología de la vía biliar; algunos son diagnósticos no invasivos y otros son invasivos con fines diagnósticos y terapéuticos. El objetivo es valorar la utilidad de la СTO en pacientes con estudio de imagen o terapéutico preoperatorio. El cirujano general debe ser consciente de las ventajas de la CTO, aun cuando se disponga de estos estudios preoperatorios.

El diagnóstico de reingreso fue lito residual y a tres de estos pacientes se les había realizado CPRE preoperatoria, dando falsos negativos (5.66\%). Un estudio con mayor muestra reporta una incidencia del 
Tabla 2. Grupos divididos según los distintos abordajes utilizados en el estudio y manejo de los pacientes

\begin{tabular}{|c|c|c|c|c|}
\hline & $\begin{array}{l}\text { CTO estudio } \\
(n=37)\end{array}$ & $\begin{array}{l}\text { CTO/no estudio } \\
\quad(n=21)\end{array}$ & $\begin{array}{l}\text { No CTO/estudio } \\
(n=54)\end{array}$ & $\begin{array}{l}\text { No CTO/no estudio } \\
(n=5)\end{array}$ \\
\hline \multicolumn{5}{|l|}{ Sexo } \\
\hline Masculino & 6 & 6 & 24 & 1 \\
\hline Femenino & 31 & 15 & 30 & 4 \\
\hline \multicolumn{5}{|l|}{ Diagnóstico } \\
\hline Coledocolitiasis & 37 & 21 & 54 & 5 \\
\hline \multicolumn{5}{|l|}{ Estudio previo } \\
\hline CPRE & 17 & - & 32 & - \\
\hline CRM & 15 & - & 2 & - \\
\hline $\mathrm{CPRE}+\mathrm{CRM}$ & 5 & - & 20 & - \\
\hline Reingreso & 0 & 1 & 3 & 5 \\
\hline \multicolumn{5}{|c|}{ Diagnóstico de reingreso } \\
\hline Lito residual & - & 1 & 3 & 5 \\
\hline
\end{tabular}

Tabla 3. Comparación entre grupos en los que se realizó o no colangiografía transoperatoria (СTO). Se observa que las tasas de reingresos en los grupos sin CTO son estadísticamente significativas comparadas con las de los grupos con СTO

\begin{tabular}{lccc}
\hline & CTO & Sin CTO & p \\
\hline Estudio previo & & & \\
Sí & $37(27.80 \%)$ & $54(38.50 \%)$ & NS \\
No & $21(25.66 \%)$ & $5(8.02 \%)$ & \\
Tipo de estudio & & & \\
CPRE & $17(14.52 \%)$ & $32(27.35 \%)$ & NS \\
CRM & $15(12.82 \%)$ & $2(1.70 \%)$ & \\
CPRE+CRM & $5(4.27 \%)$ & $20(17.09 \%)$ & \\
No estudio & $21(17.94 \%)$ & $5(4.27 \%)$ & \\
Reingreso & & & \\
Sí & $1(0.85 \%)$ & $8(6.83 \%)$ & 0.0021 \\
No & $57(48.71 \%)$ & $58(49.57 \%)$ & \\
\hline
\end{tabular}

CPRE: colangiopancreatografía retrógrada endoscópica; CRM: colangiopancreatografía por resonancia magnética; CTO: colangiografía transoperatoria; NS: no significativo.

8.3\% de falsos negativos para CPRE por sospecha de coledocolitiasis; esto dio una sensibilidad para detectar litos en la vía biliar por CPRE del 94\%, comparable a la reportada en la literatura $(95 \%)^{6,12}$. Ello puede deberse a nuevos litos que pasan a la vía biliar en el transcurso de la CPRE a la colecistectomía, una CPRE con falso negativo o una combinación de ambos factores. Es por eso que algunos grupos de cirujanos defienden la decisión de realizar una CTO de manera sistemática en estos pacientes.

El índice de falsos positivos de la CTO reportado en la literatura es del $4-24 \%{ }^{6}$. En nuestro estudio hubo un $6.25 \%$ de falsos positivos, tomando en cuenta los pacientes que reingresaron con lito residual en quienes se realizó CTO sin estudio ni tratamiento endoscópico preoperatorio.

Este estudio reportó 7 (9.45\%) CPRE fallidas, y los pacientes tuvieron que someterse a exploración de la vía biliar por no lograr resolver el cuadro obstructivo por vía endoscópica. Esta cifra coincide con lo reportado en la literatura, según la cual hasta en el $10 \%$ de los casos no se puede resolver el cuadro obstructivo mediante $\mathrm{CPRE}^{13}$. Los principales problemas que se presentan al momento de la extracción de un lito mediante CPRE son que este sea muy grande o de difícil extracción. No hay un consenso que defina un lito grande; algunos autores se refieren a lito grande en la vía biliar cuando este tiene un diámetro mayor de 10-15 mm, y otros cuando tiene un diámetro igual al del colédoco ${ }^{14}$. Por litos "difíciles» se entiende que son grandes, múltiples, intrahepáticos, en forma de barril o impactados, o cuando hay estenosis de la vía biliar o anatomía alterada ${ }^{15}$. Es importante que el cirujano general esté capacitado para poder resolver la obstrucción de la vía biliar quirúrgicamente, en caso de que no sea posible hacerlo por endoscopia ${ }^{16}$.

El 3-7\% de los pacientes con colelitiasis desarrollarán pancreatitis ${ }^{17}$, en especial tras la presencia de microlitiasis $(<5 \mathrm{~mm})$. No se recomienda realizar CPRE a los pacientes con pancreatitis biliar si no presentan datos clínicos o por imagen de obstrucción del conducto biliar. El $63-75 \%$ de estos pacientes tendrán un lito en la vía biliar en las primeras 48 horas del cuadro agudo; pasado el cuadro agudo, solo el $5 \%$ persistirán con lito en la vía biliar ${ }^{18}$. Es por esto que se recomienda realizar СТO en los pacientes con antecedente de pancreatitis biliar. Otras indicaciones para realizar СTO son por poca claridad anatómica, variantes anatómicas o por sospecha de una lesión de la vía biliar.

Los grupos que registraron el mayor número de reingresos fueron aquellos en los que no se realizó estudio o manejo endoscópico previo, representando 
el $88 \%$ de los casos que reingresaron ( $p=0.005)$, con predominio del grupo en que no se realizó estudio previo ni CTO durante el procedimiento quirúrgico $(p=0.0021)$. Los reingresos reportados en este grupo fueron por lito residual y todos se resolvieron mediante CPRE durante el reingreso, logrando resolver exitosamente el cuadro obstructivo. La incidencia de litos residuales posterior a una colecistectomía es menor del $2.5 \%{ }^{19}$. Hay distintos factores que predisponen a que esto suceda, de los cuales uno es la falla en el diagnóstico preoperatorio de coledocolitiasis y nunca resolviendo el cuadro obstructivo; otros factores son dejar el muñón del conducto cístico muy largo, colecistectomía parcial, síndrome de Mirizzi, conducto cístico con inserción baja y tejidos friables $^{19,20}$. En este estudio no se reportaron colecistectomías parciales.

Con la información obtenida se observa que los pacientes que presentaron menor índice de reingresos fueron aquellos en los que se realizó CTO durante el procedimiento quirúrgico. Los pacientes reingresados en este grupo debido a persistencia del cuadro obstructivo fueron muy pocos $(0.85 \%)$, lo cual guarda relación con la sensibilidad y la especificidad de la CTO reportadas en la literatura ${ }^{6,13-20}$.

De los tres grupos que registraron reingresos, el grupo 2 tuvo el menor porcentaje de reingresos $(0.85 \%)$. Los grupos en los que no se realizó estudio preoperatorio fueron los que más reingresos registraron $(88 \%)$.

\section{Conclusión}

Este estudio demuestra la importancia del manejo endoscópico preoperatorio en los pacientes con coledocolitiasis, así como la utilidad de complementar con CTO para detectar un lito residual.

No contar con estudio o manejo endoscópico preoperatorio es un factor que predispone a que el paciente reingrese por lito residual. Se demostró que contar con un estudio o manejo endoscópico preoperatorio y realizar CTO se asociaron a la menor tasa de reingresos en los grupos estudiados.

\section{Conflicto de intereses}

Los autores declaran no tener conflicto de intereses.

\section{Financiamiento}

No hubo fuentes de financiamiento para este trabajo.

\section{Responsabilidades éticas}

Protección de personas y animales. Los autores declaran que para esta investigación no se han realizado experimentos en seres humanos ni en animales.

Confidencialidad de los datos. Los autores declaran que han seguido los protocolos de su centro de trabajo sobre la publicación de datos de pacientes.

Derecho a la privacidad y consentimiento informado. Los autores declaran que en este artículo no aparecen datos de pacientes.

\section{Bibliografía}

1. Mirizzi PL. La cholangiografia durante las operaciones de las vías biliares. Bol Soc Cir Buenos Aires. 1932;16:1133.

2. MacFadyen BV. Intraoperative cholangiography: past, present, and future. Surg Endosc. 2006;20:436-40.

3. Chowbey P, Sharma A, Goswami A, Afaque Y, Najma K. Residual gallbladder stones after cholecystectomy: a literature review. J Min Access Surg. 2015;11:223-30.

4. Sheffield KM, Riall TS, Han Y, Kuo YF, Townsend CM Jr, Goodwin JS. Association between cholecystectomy with vs without intraoperative cholangiography and risk of common duct injury. JAMA. 2013;310:812-20.

5. Rogers SJ, Cello JP, Horn JK, Siperstein AE, Campbell AR, Campbell AR, et al. Prospective randomized trial of LC+LCBDE vs ERCP/S+LC for common bile duct stone disease. Arch Surg. 2010;145:28-33.

6. Maple JT, Ben-Menachem T, Anderson MA, Appalaneni V, Banerjee S; ASGE Standards of Practice Committee 2010. The role of endoscopy in the evaluation of suspected choledocholithiasis. Gastrointest Endosc. 2010;71:1-9.

7. Ragulin-Coyne E, Witkowski ER, Chau Z, Ng SC, Santry HP, Callery MP et al. Is routine intraperative cholangiogram necessary in the twenty-first century? A national view. J Gastrointest Surg. 2013;17:434.

8. Sirinek KR, Schwesinger WH. Has intraoperative cholangiography during laparoscopic cholecystectomy become obsolete in the era of preoperative endoscopic retrograde and magnetic resonance cholangiopancreatography? J Am Coll Surg. 2015;220:522-8.

9. Alkhaffaf B, Parkin E, Flook D. Endoscopic retrograde cholangiopancreatography prior to laparoscopic cholecystectomy: a common and potentially hazardous technique that can be avoided. Arch Surg. 2011;146:329-33.

10. Rijna H, Kemps WG, Eijsbouts Q, Meuwissen SG, Cuesta MA. Preoperative ERCP approach to common bile duct stones: results of a selective policy. Dig Surg. 2000;17:229-33.

11. Iranmanesh P, Frossard JL, Mugnier-Konrad B, Morel P, Majno P, Nguyen-Tang $T$, et al. Initial cholecystectomy vs sequential common duct endoscopic assessment and subsequent cholecystectomy for suspected gallstone migration: a randomized clinical trial. JAMA. 2014;312:137-44.

12. Borjeson J, Liu SK, Jones S, Matolo NM. Selective intraoperative cholangiography during laparoscopic cholecystectomy: how selective? Am Surg. 2000;66:616-8.

13. Richard F, Boustany M, Britt LD. Accuracy of magnetic resonance cholangiopancreatography for diagnosing stones in the common bile duct in patients with abnormal intraoperative cholangiograms. Am J Surg. 2013;205:371-3.

14. Jin PP, Cheng JF, Liu D, Mei M, Xu ZQ, Sun LM. Endoscopic papillary large balloon dilation vs endoscopic sphincterotomy for retrieval of common bile duct stones: a meta-analysis. World J Gastroenterol. 2014;20:5548-56.

15. Ozcan N, Kahriman G, Karabiyik O, Donmez H, Emek E. Percutaneous management of residual bile duct stones through T-tube tract after cholecystectomy: a retrospective analysis of 89 patients. Diagn Interv Imaging. 2017;2:149-53.

16. Pierce RA, Jonnalagadda S, Tessier DJ. Incidence of residual choledocholithiasis detected by intraoperative cholangiography at the time of laparoscopic cholecystectomy in patients having undergone preoperative ERCP. Surg Endosc. 2008;22:2365-72.

17. Acosta JM, Ledesma CL. Gallstone migration as a cause of acute pancreatitis. N Engl J Med. 1974;290:484.

18. Fogel E, Sherman S. ERCP for gallstone pancreatitis. N Engl J Med. 2014;370:150-7.

19. El Nakeeb A, Ezzat H, Askar W, Salem A, Mahdy Y. Management of residual gallbladder and cystic duct stump stone after cholecystectomy: a retrospective study. Egypt J Surg. 2016;35:391-7.

20. Ji W, Li LT, Li JS. Role of laparoscopic subtotal cholecystectomy in the treatment of complicated cholecystitis. Hepatobiliary Pancreat Dis Int. 2006;5:584-9. 